that there seems little doubt that rhapidosomes are vestiges of once complete bacteriophages and that the genomes which specify these particles are now stably associated with the genome of the host cell. Among bacteria the persistence of such vestigial virus particles and their genomes as symbionts seems to be the rule rather than the exception.

\section{CANCER RESEARCH}

\section{Tumour Vascularity}

from a Correspondent

Professor L. F. LAMERTON (Institute of Cancer Research), introducing the topic of tumour blood supply and metastasis at the ninth meeting in the principles of experimental and clinical oncology series in the British Museum (Natural History) on March 6, pointed out that the type, arrangement and morphology of blood supply are important elements in the heterogeneity of tumours, both between tumours of the same type in different individuals and within single tumours. $\mathrm{He}$ also suggested that blood supply can be a chief factor in determining the likelihood of metastases or the outcome of chemotherapy or radiotherapy. These themes were developed by the other speakers, Dr R. H. Thomlinson (Radiobiology Unit, Royal Postgraduate Medical School) and Dr M. J. Peckham (Institute of Cancer Research).

Dr Thomlinson pointed out that in different tumours the clonogenic (or proliferating) cells are found at various distances away from blood vessels, and this variation greatly affects the physiological condition of the cells and their susceptibility to attack, particularly by irradiation. In deeper regions of the tumour capillaries are likely to be attenuated and are possibly inefficient carriers of blood. There is considerable likelihood of shunts developing by anastomosis of blood yessels in the more superficial regions of the tumour. Overall blood flow through tumours tends to be slow and this can possibly be attributed to venous congestion, perhaps arising from pressure within the tumour. All too little is known about lymphatic drainage of tumours.

In discussion it came up that skin homografts without a lymphatic drainage but with an otherwise normal blood supply are not rejected. It was agreed that if the lymphatic drainage of tumours is degenerate (Dr R. J. Goldacre, Institute of Cancer Research) this could be an important factor in limiting immunological attack on a tumour. In relation to radiotherapy, in which it is known that the sensitivity of tumour cells to ionizing radiations is affected by oxygen concentration, Dr Thomlinson felt that it was important to get some idea of the pattern of re-oxygenation as the tumour mass is reduced, but it is very difficult to obtain this sort of information.

Dr Peckham described experimental studies which show that failure of development of a blood supply can limit the growth of a tumour. Nevertheless in most situations it seems that tumours have a considerable capacity to induce capillary growth, even to the extent of producing angiogenetic factors. Antibodies against these materials have been used to attempt to limit metastatic spread of tumours but so far with no success. Dr Peckham also considered the various claims that have been made that tumour cell masses are surrounded by a fibrin lattice and that this is an important factor in either tumour growth or the tendency to metastasize. He spoke of evidence that injection of fibrinolysin can reduce the incidence of metastases but said that there are various and partly conflicting results. In similar experiments it has been shown that warfarin increases the prothrombin time to two to three times normal and there can also be a reduced incidence of metastases. Here, however, it is difficult to exclude a direct effect of the warfarin on populations of tumour cells.

The meeting revealed that although tumour vascularity and the relationship of the tumour to the connective tissue materials in which it grew are topics of considerable import the complexities of the situation have tended to defy useful analysis.

\section{BIOPOLYMERS}

\section{The End of the Tether}

from our Molecular Biology Correspondent THE notion of tethering biochemically interesting molecules, such as enzymes, to insoluble matrices by way of covalent bonds can scarcely be seen as a new and revolutionary insight. The first successful attempts at such a strategy with a view to controlling the exposure of substrates to enzymes, and for isolating biologically active species by affinity methods, were indeed described many years ago, but it is only really in the past year or two that this general approach seems to have become lodged in the collective subconscious of the biochemical cohorts. In this time it has without doubt brought about a minor revolution in biochemical practice, and the scope of the applications is still widening, partly at least under the stimulus of various technical improvements. Some interesting examples are to be found in the current literature.

One area that has been completely transformed by the matrix attachment approach is that of peptide synthesis, the efficiency of which has now reached a level that would have been unthinkable in the days when the product after each addition had to be recovered from solution. The efficiency of the method is limited by the extent to which any addition cycle falls short of complete reaction, and this allows truncated or incorrect chains to be formed in appreciable amounts. Bayer et al. (J.Amer. Chem. Soc., 94, 265; 1972)

\title{
Alternation of DNA Conformations
}

IN next Wednesday's Nature New Biology (March 29) Pilet and Brahms report a reinvestigation of the $B \rightleftharpoons A$ transition of DNA, in relation to its base composition. The B form was first recognized at high humidities, and it is presumed to represent the state of DNA in solution. The bases are tilted relative to the helix axis, whereas in the A form the bases lie perpendicular to the axis. The precise humidity at which the A form appears depends critically on the salt concentration. At lower humidities denaturation occurs and the ordered structure is lost. Because of the difference in the tilt of the bases, and accompanying changes in the orientation of the phosphate groups, it is possible to distinguish the $A$ and $B$ forms by measurements of infrared dichroism of oriented films.

Pilet and Brahms have observed the $B \rightleftharpoons A$ transition by this means and find that in typical DNA of relatively high $(\mathrm{G}+\mathrm{C})$ content, the change occurs within a very narow humidity range. They find, however, that the lower the $(\mathrm{G}+\mathrm{C})$ content the more reluctant the A form is to appear. In the extreme case of crab satellite DNA, which contains less than 5 per cent of $(G+C)$ and is essentially an alternating polymer of $A$ and $\mathrm{T}$, the $\mathrm{A}$ form does not appear at all, the $B$ structure giving place directly to the denatured state, the unfolding beginning below 90 per cent relative humidity.

Pilet and Brahms have constructed a phase diagram indicating the ranges of stability of the A, B and denatured forms of DNA as a function of base composition and relative humidity; from this it seems that if the $(G+C)$ content falls below about 35 per cent the zone of stability of the A form disappears. The possibility that inhomogeneous distribution of base composition along a DNA will give rise to an alternation of conformations within a single molecule must be considered. 